\title{
Caustic activation of rain showers
}

\author{
Michael Wilkinson ${ }^{1}$, Bernhard Mehlig ${ }^{2}$ and Vlad Bezuglyy ${ }^{2}$ \\ ${ }^{1}$ Faculty of Mathematics and Computing, \\ The Open University, Walton Hall, \\ Milton Keynes, MKY 6AA, England \\ ${ }^{2}$ Department of Physics, Göteborg University, \\ 41296 Gothenburg, Sweden
}

\begin{abstract}
We show quantitatively how the collision rate of droplets of visible moisture in turbulent air increases very abruptly as the intensity of the turbulence passes a threshold, due to the formation of fold caustics in their velocity field. The formation of caustics is an activated process, in which a measure of the intensity of the turbulence, termed the Stokes number St, is analogous to temperature in a chemical reaction: the rate of collision contains a factor $\exp (-C / \mathrm{St})$. Our results are relevant to the long-standing problem of explaining the rapid onset of rainfall from convecting clouds. Our theory does not involve spatial clustering of particles.

PACS numbers: $45.50 \mathrm{Tn}, 47.55 \mathrm{D}, 92.60 \mathrm{Mt}, 92.60 \mathrm{Nv}$
\end{abstract}

It is common experience that rainfall can commence very abruptly from cumulus clouds (which form when the atmosphere is convecting) but has a much slower onset from stratiform clouds in a stable atmosphere. This can happen even when no part of the cloud is below freezing point. It is believed that the difference between convecting and stable clouds arises because the convection gives rise to small-scale turbulent motion which facilitates the coalescence of microscopic water droplets ('visible moisture') into raindrops. This idea has a long history ([1] is a significant contribution containing references to other early papers), but a satisfying theory has been elusive and the topic remains a subject of intensive research, reviewed recently in 22. Numerical experiments have shown a dramatic increase in the rate of collision of suspended particles when the intensity of turbulence exceeds a certain threshold. This effect was first described in [3], see also [4]. Here we present a simple quantitative theory of this phenomenon, illustrated in figure 1 which shows the collision rate $R$ of an aerosol as a function of a dimensionless parameter termed the Stokes number, St, which contains information about the turbulence intensity, $\epsilon$, and the radius of the water droplets, $a$ (other symbols in the caption are defined later). There is a precipitous increase in the collision rate at a threshold value of St, which was also observed in 3, 4]. The current consensus, represented in [2, 3, 4, [5], is that the increased rate of collision involves spatial clustering of particles. One exception is [6], which presents a theory having elements (described later) in common with our own, but which is more complex in its formulation and less precise in its conclusions. Spatial clustering plays no role in our theory.

The properties of clouds are very variable and the sizes of visible moisture droplets have a large dispersion, but typically the average of the radius is approximately $10 \mu \mathrm{m}$ and the density is $n \approx 10^{8} \mathrm{~m}^{-3}[2]$. The motion of the droplets is dominated by viscous forces, so that the equation for the position $\boldsymbol{r}$ of a droplet is well approximated by

$$
\ddot{\boldsymbol{r}}=\gamma(\boldsymbol{u}(\boldsymbol{r}, t)-\dot{\boldsymbol{r}})
$$

until the particles come into contact [1] (here $\boldsymbol{u}(\boldsymbol{r}, t)$ is the velocity field of the air and dots denote derivatives with respect to time). According to Stokes's formula for the viscous drag on a sphere, $\gamma=9 \rho_{\mathrm{g}} \nu / 2 \rho_{\mathrm{f}} a^{2}$ ( $\nu$ being the kinematic viscosity of air, $\rho_{\mathrm{f}}$ and $\rho_{\mathrm{g}}$ the densities of water and air respectively): using the values above we estimate $\gamma \approx 500 \mathrm{~s}^{-1}$. The turbulent motion is a multiscale flow with an approximately power-law spectrum of spatial fluctuations of $\boldsymbol{u}(\boldsymbol{r}, t)$ 7]. According to the Kolmogorov theory of turbulence, the short wavelength cutoff of the power-law spectrum, $\eta$ (termed the Kolmogorov length) is a function of $\nu$ and of the rate of dissipation

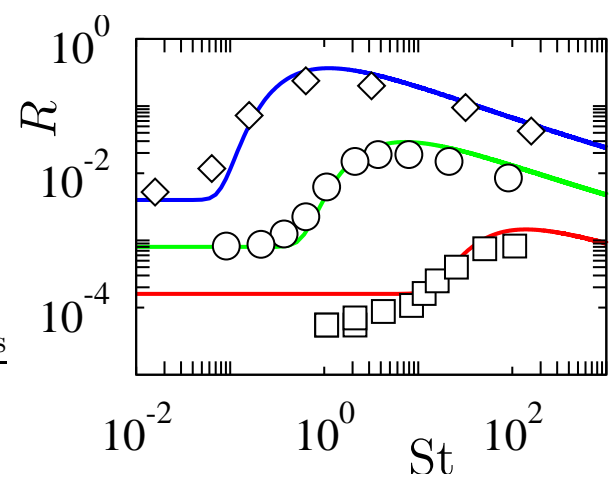

FIG. 1: Particle collision rate as a function of Stokes number, for three different values of the Kubo number (defined in equation (3)). In these simulations we used $n=10^{3}$, $a=2.5 \times 10^{-4}, \eta=0.1 / \pi, \tau=0.1$, and varied $u_{0}$ and $\gamma$ : $\mathrm{Ku}=1(\diamond), \mathrm{Ku}=0.2(\circ)$, and $\mathrm{Ku}=0.04(\square)$. The theoretical curves are (17), with actions $S$ obtained from figure 3 The constant $C_{\mathrm{a}}$ in $R_{\mathrm{a}}=C_{\mathrm{a}} n a^{d} u_{0} / \eta$ was fitted (see text). 
per unit mass in the turbulence, $\epsilon$. Similar considerations apply to the shortest characteristic timescale, $\tau$. Dimensional arguments [7] then imply

$$
\eta \sim\left(\nu^{3} / \epsilon\right)^{1 / 4}, \quad \tau \sim(\nu / \epsilon)^{1 / 2} .
$$

The typical velocity difference for points separated by the correlation length $\eta$ will be denoted by $u_{0}$. The dimensional arguments of the Kolmogorov theory imply that $u_{0} \sim \eta / \tau$ for turbulent flow. The dissipation rate $\epsilon$ is highly variable between different clouds. Values of $\epsilon=0.1 \mathrm{~m}^{3} \mathrm{~s}^{-2}$ are typical [2]; $\nu \approx 10^{-5} \mathrm{~m}^{2} \mathrm{~s}^{-1}$ for air in the lower atmosphere, giving $\eta \approx 3 \times 10^{-4} \mathrm{~m}, \tau \approx 10^{-2} \mathrm{~s}$.

We can form four independent dimensionless parameters describing the microscopic motion of the water droplets: these are

$$
\mathrm{St}=\frac{1}{\gamma \tau}, \quad \mathrm{Ku}=\frac{u_{0} \tau}{\eta}, \quad n \eta^{d}, \quad \eta / a
$$

( $d$ is the dimensionality of space). The values quoted above give $\mathrm{St} \approx 0.2$. The Kubo number $\mathrm{Ku}$ cannot be large [9] and the comments above indicate that it is of order unity for fully developed turbulence, but small values of $\mathrm{Ku}$ occur in stirred fluids, and this parameter will play a role later. From the data above we see that $n \eta^{d} \ll 1$ and $\eta / a \gg 1$ for water droplets in clouds.

Saffman and Turner [1] only considered the case of St $\ll 1$ in detail, because they believed that St $\gg 1$ would only be realised in the most unstable cumulonimbus clouds. Later it was understood that fully-developed turbulent motion with high Reynolds number can exhibit pronounced intermittency [7]. One consequence is that the rate of dissipation can fluctuate wildly relative to the mean value, and measurements in clouds have indicated that $\epsilon$ can have an approximately log-normal distribution [2]. The notion that intermittency can promote the formation of rain showers by creating localised regions of very high turbulent intensity is considered in [2] and [6].

Aerosol particles in a turbulent flow may exhibit a degree of clustering: mechanisms for this process were proposed in [5, 6, 8] and reference [9] describes recent advances. Most of the recent literature on the initiation of rainfall by turbulence assumes that the mechanism involves clustering 2, 3, 4, 5]. This theory is unsatisfactory for a number of reasons. Firstly, the widely accepted view is that clustering is due to particles being centrifuged away from regions of high vorticity. It is argued that this effect is strongest when St is close to unity: it does not occur when the particle motion is highly damped $(\mathrm{St} \ll 1)$, or when the vortices are too short-lived (St $\gg 1)$. Figure 1 shows, however, that the collision rate rises abruptly at a threshold value of $\mathrm{St}$ (which depends upon $\mathrm{Ku}$ ) and remains high for $\mathrm{St} \gg 1$ : this is equally true for simulations of single-scale flows, such as figure 1 and multi-scale flows, such as those in [3]. Another difficulty is that the clustering can only occur for particle separations smaller than $\eta$, but in a cloud the density of particles is so low that there is unlikely to be more than one particle in a cube of length $\eta$. Also, it has not been established that the centrifuge mechanism is sufficiently effective to explain the large increase in collision rate.

In our theory, the dramatic increase in the collision rate is a consequence of the formation of 'caustics', illustrated schematically in figure 2 Here the velocity of the water droplets as a function of position is initially singlevalued (curve (a)) but droplets with a large velocity overtake slower-moving particles, so that at a later time the droplet velocity is multi-valued (curve (b)). The region where the velocity is a multi-valued function is bounded by two fold caustics. The caustics have two effects which could enhance the collision rate of water droplets. Firstly, at the caustic lines themselves there can be a divergence in the density of particles. This effect is discussed in [6, 10]: it is analogous to the divergence of light intensity on optical caustics 11], but we argue that it is not relevant to collisions between microscopic water droplets in clouds, where $n \eta^{3} \ll 1$. The other effect of the caustics is that when the velocity field is multi-valued, droplets at the same position are moving with differing velocity, and their relative motion produces collisions. This has no analogue in optical caustics. The importance of this effect was previously emphasised in [6].

Our theory must consider the rate of collision between droplets both with and without caustics, and the rate at which caustics are formed. Saffman and Turner [1] discussed the collision (due to shearing motion) of droplets which are advected with the air: this approximation is valid in the limit as $\mathrm{St} \rightarrow 0$; we term this advective collision rate $R_{\mathrm{a}}$. At large Stokes numbers a theory due to Abrahamson [12] treats the water droplets as a gas of particles with random and uncorrelated motion and calculates the collision rate $R_{\mathrm{g}}$ by gas-kinetic theory. This theory is widely regarded as being applicable only at very large Stokes numbers, and indeed the two theories give results which differ by a factor of order $\eta / a$ at $\mathrm{St} \approx 1$. This might be taken as evidence that at least

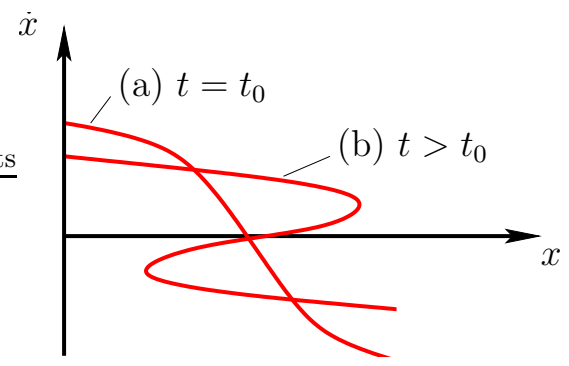

FIG. 2: Schematic showing the formation of a caustic: (a) Initial configuration, showing manifold representing droplet velocity as a function of position. (b) This manifold has developed fold caustics. The velocity field is multivalued between the folds. 
one of these theories does not work when the Stokes number is of order unity. However, we shall argue that the gas kinetic model is applicable as soon as caustics have formed and that the collision rate is well-approximated by $R=R_{\mathrm{a}}+f(\mathrm{St}, \mathrm{Ku}) R_{\mathrm{g}}$, where $f(\mathrm{St}, \mathrm{Ku})$ is the fraction of the coordinate space for which the velocity field has become multi-valued due to the formation of caustics.

First we consider the rate of production of caustics in more depth. In earlier works $([10,13])$, two of the present authors described the formation of caustics in the limit where $\mathrm{Ku} \ll 1$. In the one-dimensional case, we considered the linearisation of equation (1), describing the small separation in space $\delta x$ and velocity $\delta v$ for two nearby droplets. The equation of motion for $X=\delta v / \delta x$ is 14]

$$
\frac{\mathrm{d} X}{\mathrm{~d} t}=-\gamma X-X^{2}+\frac{\partial u}{\partial x}(x(t), t)
$$

which is a stochastic differential equation, in which the velocity gradient acts as a random forcing term. The droplets encounter a caustic whenever $\delta x$ passes through zero, implying that $X$ goes to infinity in one direction, then jumps instantaneously to the reflected point at infinity. When fluctuations drive $X$ to a sufficiently negative value, it will almost certainly escape to $-\infty$ in a finite time. In [13], equation (44) was considered in the limit St $\rightarrow \infty, \mathrm{Ku} \rightarrow 0$, where the dynamics of $X$ can be approximated by a Langevin process. The associated Fokker-Planck equation was solved exactly, giving the rate $J$ at which any one droplet passes through caustics. Similar results were obtained in two [10] and three [9] spatial dimensions. They are expressed in terms of a dimensionless parameter $\mathcal{I}$ defined in terms of the strainrate correlation function

$$
\mathcal{I}=\frac{1}{2 \gamma} \int_{-\infty}^{\infty} \mathrm{d} t\left\langle\frac{\partial u_{1}}{\partial x_{1}}(\boldsymbol{r}(t), t) \frac{\partial u_{1}}{\partial x_{1}}(\boldsymbol{r}(0), 0)\right\rangle
$$

(we use angular brackets to indicate averages). In the limit as $\mathcal{I} \rightarrow 0$, we find that the rate of caustic formation is determined by the rate of escape due to diffusion of the coordinate $X$ from an attractive fixed point at $X=0$. The escape rate is asymptotic to

$$
J=J_{0} \exp (-S / \mathcal{I})
$$

(for some constant $J_{0}$ ), where in $S$ can be obtained as the action of a trajectory of a Hamiltonian function [15, 16]. Note that we can write $\mathcal{I} \sim \mathrm{Ku}^{2} \mathrm{St}$, so that (6) is consistent with the alternative form quoted in the abstract. In one dimension we extract $S=1 / 6$ from our exact expression for $J$, obtained in [13]. In two and three dimensions, one obtains $S \approx 0.14[10]$ and $S \approx 0.12[\underline{9}]$ in the limit as $\mathrm{Ku} \rightarrow 0$. Our numerical results, summarised in figure 3 show that the activated escape model for the formation of caustics, described by (6), is valid even when $\mathrm{Ku}$ approaches unity. We find that the action $S$ depends upon
Ku. For our model (described below) we found $S=0.70$ for $\mathrm{Ku}=1, S=0.18$ for $\mathrm{Ku}=0.2$ and $S=0.15$ for $\mathrm{Ku}=0.04$, consistent with the limiting value $S=0.14$ for $\mathrm{Ku} \rightarrow 0$ quoted above.

The dependence of $J$ upon $\mathcal{I}$ (or equivalently, on St) is analogous to the dependence of the rate of a chemical reaction on temperature $T$, which is well approximated by expressions containing an Arrhenius factor, $\exp (-E / k T)$ ( $E$ is the activation energy, $k$ is the Boltzmann constant). In this sense we may regard the formation of caustics as an activated process, in which the dimensionless intensity of the turbulence $(\mathcal{I}$ or St) plays a role analogous to temperature.

We argue that the function $f(\mathrm{St}, \mathrm{Ku})$ describing the prevalence of caustics is closely related to the rate of caustic formation, so that the collision rate is well approximated by

$$
R=R_{\mathrm{a}}+\exp (-S / \mathcal{I}) R_{\mathrm{g}} .
$$

Formulae for the advective and gas-kinetic collision rates, $R_{\mathrm{a}}$ and $R_{\mathrm{g}}$ respectively, are given below. It is equation (7) which is the theoretical curve illustrated in figure 1 using values of $S$ obtained from figure 3 We fitted the prefactor of the expression for $R_{\mathrm{a}}$, which is the dominant term when St $\ll 1$, but there are no other fitted parameters. We remark that combining equations (2) and (4) of [6] gives an expression which can be written in the form $R=R_{\mathrm{a}}+P R_{\mathrm{c}}$, analogous to our (7), in which $R_{\mathrm{c}}$ is similar to the precise asymptote, $R_{\mathrm{g}}$. The expression for the factor $P$ in $[\underline{6}]$ has a different dependence upon St.

Our numerical simulations use a two-dimensional model in which the divergenceless velocity $\boldsymbol{u}(\boldsymbol{x}, t)$ is obtained from a scalar random field $\psi(\boldsymbol{x}, t)$ by writing $\boldsymbol{u}=(\partial \psi / \partial y,-\partial \psi / \partial x)$. The field $\psi(\boldsymbol{x}, t)$ is a Gaussian random function with spatially and temporally stationary, isotropic statistics. The mean value is zero and the correlation function is $\left\langle\psi(\boldsymbol{x}, t) \psi\left(\boldsymbol{x}^{\prime}, t^{\prime}\right)\right\rangle=$

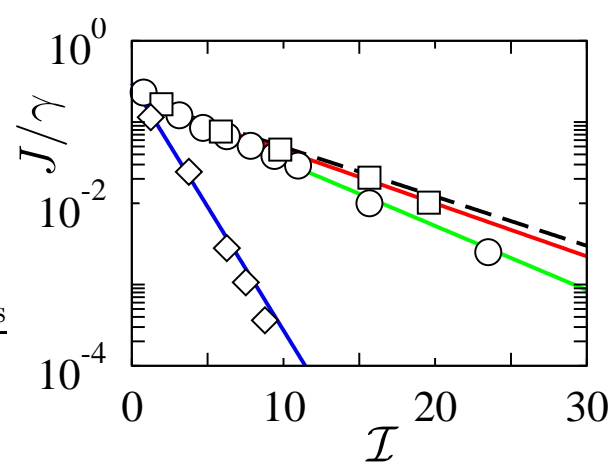

FIG. 3: Rate of caustic formation as a function of $\mathcal{I} \propto \mathrm{Ku}^{2} \mathrm{St}$, showing evidence for 'activated' behaviour: $\mathrm{Ku}=1(\diamond)$, with action $S=0.70, \mathrm{Ku}=0.2, S=0.18$ (०), and $\mathrm{Ku}=0.04$, $S=0.15(\square)$. Also shown is the limiting behaviour as $\mathrm{Ku}$ $\rightarrow 0$, with action $S=0.14$ (dashed line). 
$u_{0}^{2} \exp \left[-\left(t-t^{\prime}\right)^{2} / 2 \tau^{2}\right] \exp \left(-\left|\boldsymbol{x}-\boldsymbol{x}^{\prime}\right|^{2} / 2 \eta^{2}\right)$, which gives $\mathcal{I}=\sqrt{\pi / 2} \mathrm{Ku}^{2} \mathrm{St}$ for this model. The droplets are initially randomly positioned, and they are regarded as having collided when their separation falls below $2 a$ (that is, we assume that the collision efficiency is unity [1]). Figure 1 shows the rate of collision $R$ for a single particle. We did not include gravitational settling or other effects which also occur without turbulence. The results for our single-scale flow are very similar to the simulations using Navier-Stokes flows, reported in [3, 4], implying that the multi-scale aspect of those simulations is not essential to their understanding.

In order to complete the discussion of our formula (7) for the collision rate we describe the asymptotic formulae for the advective and gas-kinetic collision rates. In the advective limit the suspended particles are brought into contact by the effect of shearing motion in the flow. The typical shear rate is $u_{0} / \eta$. The relative velocity for particles that will be brought into contact is $\Delta v \sim a u_{0} / \eta$, and the volume swept in time $\Delta t$ is $\Delta V \sim a^{d} u_{0} \Delta t / \eta$. Collisions typically occur when $n \Delta V \sim 1$, so that the expected rate of collision is $R_{\mathrm{a}}=C_{\mathrm{a}} n a^{d} u_{0} / \eta$ (where $C_{\mathrm{a}}$ is a constant which we fitted numerically). Saffman and Turner [1] gave an upper bound on the collision rate which can be calculated analytically if correlation functions of the flow field are known. Their approach gives a precise asymptote for the steady hyperbolic flow which they discussed, but in general it is an upper bound because their calculation does not account for multiple collisions in generic flows (which are not steady and which may be locally elliptic).

When St $\gg 1$, the inertia of the aerosol particles means that their motion becomes uncorrelated with the velocity of fluid, and a gas-kinetic model of the type proposed by Abrahamson 12] is appropriate. The maximal Lyapunov exponent $\lambda$ for motion of the particles is positive 15] and if the rate of collision satisfies $R \ll \lambda$, then the velocities of nearby particles are completely randomised by the time they collide (our estimates below verify this assumption). The aerosol particles become a gas of droplets with velocities which are uncorrelated with each other and with that of the turbulent air. The velocity distribution is Maxwellian, even if $\boldsymbol{u}$ is not Gaussian distributed, because the equation of motion for the droplet velocity is analogous to an Ornstein-Uhlenbeck process. The rate of collision is exactly the same as for a hard-sphere gas with the same particle radius and r.m.s. velocity. The number of collisions per unit time of a given particle in a dilute gas is $R_{\mathrm{g}} \sim n a^{d-1} \bar{v}_{\mathrm{r}}\left(R_{\mathrm{g}}=4 a n \bar{v}_{\mathrm{r}}\right.$ in two dimensions), where $\bar{v}_{\mathrm{r}}$ is the mean relative speed of the droplets. For a Maxwellian velocity distribution, $\bar{v}_{\mathrm{r}}=\sqrt{\pi / 4} \sqrt{\left\langle\boldsymbol{v}_{\mathrm{r}}^{2}\right\rangle}$ in two dimensions and we have $R_{\mathrm{g}}=2 \sqrt{\pi} n a \sqrt{\left\langle\boldsymbol{v}_{\mathrm{r}}^{2}\right\rangle}$. It remains to calculate the r.m.s. velocity of the water droplets, $\left\langle\boldsymbol{v}^{2}\right\rangle$, from which we obtain $\left\langle\boldsymbol{v}_{\mathrm{r}}^{2}\right\rangle=2\left\langle\boldsymbol{v}^{2}\right\rangle$. The solution of the equation of motion (1) for the velocity of a droplet is

$$
\boldsymbol{v}(t)=\gamma \int_{-\infty}^{t} \mathrm{~d} t^{\prime} \exp \left[-\gamma\left(t-t^{\prime}\right)\right] \boldsymbol{u}\left(\boldsymbol{r}\left(t^{\prime}\right), t^{\prime}\right)
$$

The variance of the velocity is obtained by squaring this expression and averaging: when St $\gg 1$ this is asymptotic to

$$
\left\langle\boldsymbol{v}^{2}\right\rangle=\frac{\gamma}{2} \int_{-\infty}^{\infty} \mathrm{d} t\langle\boldsymbol{u}(\mathbf{0}, t) \cdot \boldsymbol{u}(\mathbf{0}, 0)\rangle
$$

This gives a collision rate $R_{\mathrm{g}} \sim n a^{d-1} u_{0} \sqrt{\gamma \tau}$, (for our two-dimensional model, $R_{\mathrm{g}}=2^{7 / 4} \pi^{3 / 4} \operatorname{nau}_{0} \sqrt{\gamma \tau}$ ). Thus $R_{\mathrm{g}} / R_{\mathrm{a}}$ is of order $\eta / a$ when $\mathrm{St} \approx 1$ and $\mathrm{Ku} \approx 1$.

In summary, we have shown that the collision rate is well approximated by the gas-kinetic model as soon as the turbulence intensity $\mathcal{I}$ exceeds the action $S$. Finally, we must consider whether this gives a sufficiently rapid rate of collision. In three dimensions, the gas-kinetic model gives a collision rate $R_{\mathrm{g}}=16 \sqrt{\pi / 3} n a^{2} \sqrt{\left\langle\boldsymbol{v}^{2}\right\rangle}$. From (9), we estimate $\left\langle\boldsymbol{v}^{2}\right\rangle \sim \sqrt{\mathrm{St}} \eta / \tau$. Using the above data, this estimate gives a collision rate of $R_{\mathrm{g}} \approx 5 \times 10^{-3} \mathrm{~s}^{-1}$ when $\mathrm{St} \approx 1$. We conclude that rainfall can be initiated in a timescale of a few minutes, provided that a sufficiently large part of the cloud has a turbulence intensity, $\mathcal{I}$ which exceeds the action for forming caustics, $S$.

B. M. acknowledges support by Vetenskapsrådet.

[1] P. G. Saffman and J. S. Turner, J. Fluid Mech., 1, 16-30, (1956).

[2] R. A. Shaw, Annu. Rev. Fluid Mech., 35, 183-227, (2003).

[3] S. Sundaram and L. R. Collins, J. Fluid Mech., 335, 75109, (1997).

[4] Y. Zhou, A. S. Wexler and L.-P. Wang, Phys. Fluids, 10, 1206-16, (1998).

[5] M. R. Maxey, J. Fluid Mech., 174, 441, (1987).

[6] G. Falkovich, A. Fouxon and G. Stepanov, Nature, 419, 151-154, (2002).

[7] U. Frisch, Turbulence, Cambridge University Press, (1997).

[8] J. Sommerer and E. Ott, Science, 259, 335, (1993).

[9] K. Duncan, B. Mehlig, S. Östlund and M. Wilkinson, Phys. Rev. Lett., 95, 240602, (2005).

[10] M. Wilkinson and B. Mehlig, Europhysics Lett., 71, 18692, (2005).

[11] M. V. Berry, Singularities in Waves, Les Houches Lecture Series Session XXXV, eds. R. Balian, M. Kléman and JP. Poirier, North Holland: Amsterdam, 453-543, (1981).

[12] J. Abrahamson, Chem. Eng. Sci., 30, 1371-9, (1975).

[13] M. Wilkinson and B. Mehlig, Phys. Rev. E, 68, 040101, (2003).bbbbb

[14] L. Piterbarg, SIAM J. Appl. Math., 62, 777-800, (2001).

[15] B. Mehlig and M. Wilkinson, Phys. Rev. Lett., 92, 250602, (2004).

[16] M. I. Freidlin and A. D. Wentzell, Random Perturbations of Dynamical Systems, Springer, New York, (1984). 